\title{
Functional assessment of the V390F mutation in the CCT $\delta$ subunit of chaperonin containing tailless complex polypeptide 1
}

\author{
Josefine Vallin ${ }^{1} \cdot$ Julie Grantham ${ }^{1}$ (D) \\ Received: 8 June 2021 / Revised: 19 August 2021 / Accepted: 7 September 2021 / Published online: 15 October 2021 \\ (C) The Author(s) 2021, corrected publication 2022
}

\begin{abstract}
The chaperonin containing tailless complex polypeptide 1 (CCT) is a multi-subunit molecular chaperone. It is found in the cytoplasm of all eukaryotic cells, where the oligomeric form plays an essential role in the folding of predominantly the cytoskeletal proteins actin and tubulin. Both the CCT oligomer and monomeric subunits also display functions that extend beyond folding, which are often associated with microtubules and actin filaments. Here, we assess the functional significance of the CCTS V390F mutation, reported in several cancer cell lines. Upon transfection into B16F1 mouse melanoma cells, GFP-CCT $\delta^{\mathrm{V} 390 \mathrm{~F}}$ incorporates into the CCT oligomer more readily than GFP-CCT $\delta$. Furthermore, unlike GFP-CCT $\delta$, GFP$\mathrm{CCT} \delta^{\mathrm{V} 390 \mathrm{~F}}$ does not interact with the dynactin complex component, $\mathrm{p} 150^{\mathrm{Glued}}$. As CCT $\delta$ has previously been implicated in altered migration in wound healing assays, we assessed the behaviour of GFP-CCT $\delta^{\mathrm{V} 390 \mathrm{~F}}$ and other mutants of CCT 8 , previously used to assess functional interactions with $\mathrm{p} 150^{\mathrm{Glued}}$, in chemotaxis assays. We developed the assay system to incorporate a layer of the inert hydrogel GrowDex ${ }^{\circledR}$ to provide a 3D matrix for chemotaxis assessment and found subtle differences in the migration of $\mathrm{B} 16 \mathrm{~F} 1$ cells, depending on the presence of the hydrogel.
\end{abstract}

Keywords Molecular chaperone $\cdot$ Cancer cell line $\cdot$ Chemotaxis $\cdot$ GrowDex ${ }^{\circledR}$

\section{Introduction}

Chaperonin containing tailless complex polypeptide 1 (CCT) is a molecular chaperone that promotes the folding of substrates in an ATP-dependent manner. In its oligomeric state, CCT is composed of two rings stacked back-to-back where each ring contains eight individual subunits (named $\alpha-\theta$ or $1-8)$, each occupying a fixed position within the ring (reviewed by Vallin and Grantham 2019). The eight CCT subunit genes are essential in yeast (reviewed by Stoldt et al. 1996), and CCT is found in the cytoplasm of all eukaryotes. All CCT subunits share a common domain architecture, consisting of a highly conserved equatorial domain containing an ATP-binding pocket, a less conserved apical substrate binding domain and a linker domain.

The CCT oligomer folds the cytoskeletal proteins actin and tubulin, which are both dependent on CCT to reach their final native structure (Sternlicht et al. 1993) and its

Julie Grantham

julie.grantham@cmb.gu.se

1 Department of Chemistry and Molecular Biology, University of Gothenburg, 40530 Gothenburg, Sweden folding activity also extends to other proteins, although not all are considered to be obligate folding substrates (Willison 2018). In addition to folding, CCT oligomer has functions that can be connected to assembled cytoskeletal structures, for example, by interacting with and potentially sequestering the actin filament severing protein gelsolin (Brackley and Grantham 2011; Svanstrom and Grantham 2016). Furthermore, the CCT oligomer binds to the transcription factor STAT3, potentially modulating STAT3 phosphorylation (Vallin et al. 2021).

The CCT oligomer is dynamic with regard to assembly (Roobol et al. 1999a), and in addition to CCT existing as an oligomer, it can also be detected as smaller assemblies (Collier et al. 2021; Liou and Willison 1997) and as a pool of free monomers. Equivalent mutants in the ATP-binding site of all eight CCT subunits give rise to different phenotypes in yeast consistent with the possibility of CCT subunits having distinct monomer functions (Amit et al. 2010). Furthermore, in yeast, a non-stoichiometric level of CCT subunits is observed, also consistent with at least some CCT subunits being available in increased levels as monomers (Matalon et al. 2014). For some CCT subunits, individual monomeric functions have indeed been established, for example, in cell 
lysates, CCT $\alpha, \gamma, \zeta$, and $\theta$ have been shown to co-assemble with microtubules (Roobol et al. 1999b). Furthermore, monomeric CCT $\varepsilon$ can interact with the co-transcriptional activator of the serum response factor pathway, MRTF-A (Elliott et al. 2015), and CCT $\delta$ can localize to the plasma membrane via interactions with $\mathrm{p} 150^{\text {Glued }}$, a component of the dynactin complex (Echbarthi et al. 2018).

CCT levels are known to be upregulated in hepatocellular and colonic carcinoma samples (Yokota et al. 2001) and in other human tumour samples (The Cancer Genome Atlas TCGA). CCT2 (CCT $\beta$ ) has also been implicated in cell growth and invasion (Showalter et al. 2020) and depletion of CCT $\beta$, СCT $\delta$, or СCT $\zeta$ by siRNA lead to a reduction in growth (Grantham et al. 2006). In addition, the gene expression of CCT $\delta$ and CCT $\gamma$ subunits has been shown to be increased in cells from a primary tumour migrating into a needle of extracellular matrix (Wang et al. 2004). As gene expression will be deregulated in tumours, changes to the CCT subunit levels will have the potential to lead to changes in the availably of CCT subunits both for assembly/folding and for individual CCT subunit monomer functions. Here, we study the point mutation V390F in CCT8, which occurs in five human cancer cell lines: carcinomas of the oesophagus, ovary, stomach, aerodigestive tract, and haematopoietic/ lymphoid neoplasm (reported in COSMIC) and is located at the border between the intermediate and apical domain. We characterize this mutation using the mouse melanoma $\mathrm{B} 16 \mathrm{~F} 1$ cell line and assess the potential impact of $\mathrm{CCT} \delta^{\mathrm{V} 390 \mathrm{~F}}$ upon CCT oligomer assembly, interactions with $\mathrm{p} 150^{\mathrm{Glued}}$, and in chemotaxis migration.

\section{Results and discussion}

\section{Enhanced incorporation of GFP-CCT $\delta^{\mathrm{V} 390 \mathrm{~F}}$ into the CCT oligomer}

The $\mathrm{CCT} \delta^{\mathrm{V} 390 \mathrm{~F}}$ mutation is located at the border between the apical and intermediate domains (Fig. 1a) and is shown mapped onto the structure of the alpha thermosome chain (PDB: 1A6D) (Fig. 1b). This region of sequence is identical between human and mouse CCT (Fig. 1c). The corresponding position is well conserved between orthologues, and comparison of subunits within the same species shows this position in other subunits to be occupied by leucine, isoleucine, or valine; all hydrophobic and non-polar amino acids (Sup. Figure 1a and b).

We assessed if the V390F substitution could have an impact upon CCT oligomer assembly in $\mathrm{B} 16 \mathrm{~F} 1$ mouse melanoma cells. To this end, we transfected cells with either CCT $\delta$ or $\mathrm{CCT}^{\mathrm{V} 390 \mathrm{~F}}$ (using the mouse sequence) as GFP fusions or with a myc-tag. Fusing a GFP-tag to the N-terminus of CCT subunit constructs has been shown previously to hinder

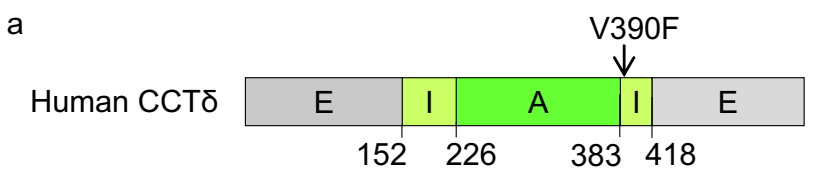

b

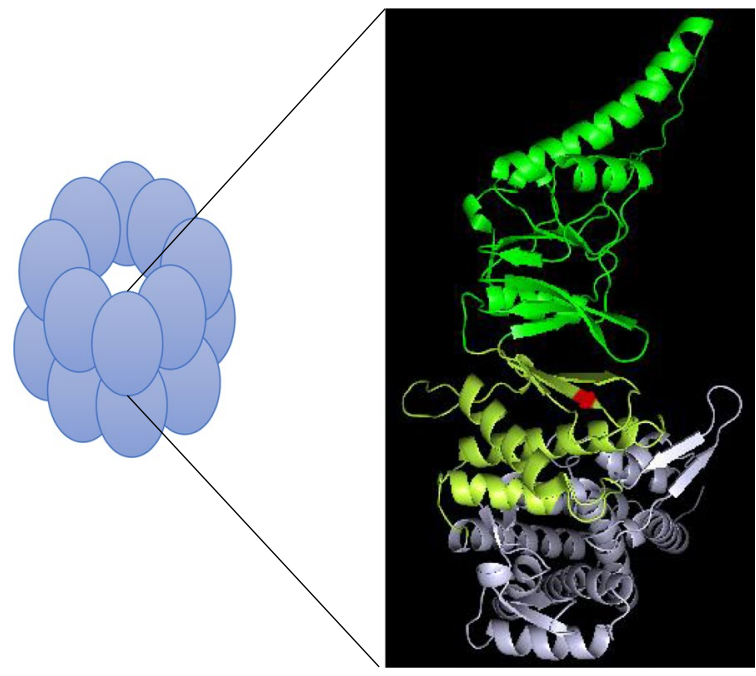

Position

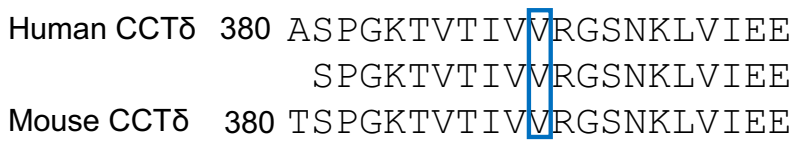

Fig. 1 Position of the highly conserved $\mathrm{CCT} \delta^{\mathrm{V} 390}$. a Cartoon representation of the CCT domains (grey E equatorial, yellow I intermediate, green A apical) with domain boundaries and V390 indicated. b Cartoon of the CCT oligomer and the structure of the thermosome alpha chain (PDB: 1A6D) are shown where the domains are colourcoded as in a and the position of V390 indicated in red. c Sequence alignment of human and mouse CCT $\delta$ in the region close to V390

incorporation into the CCT oligomer (Spiess et al. 2015). However, a C-terminal myc-tag appears to be more tolerated with regard to assembly (Brackley and Grantham 2010).

Lysates from transfected B16F1 cells were analyzed by sucrose density gradient fractionation to separate the CCT oligomer from monomeric subunits after transfection with constructs with either a GFP fusion or a myc-tag

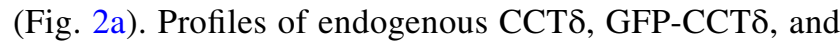
GFP-CCT $\delta^{\mathrm{V} 390 \mathrm{~F}}$ were then analyzed by Western blotting (Fig. 2b). For GFP-CCT $\delta$ and GFP-CCT ${ }^{\mathrm{V} 390 \mathrm{~F}}$, the fractions corresponding to the position of the CCT oligomer were further analyzed by immunoprecipitating the GFP fusions with anti-GFP nanobodies. In both cases, endogenous CCT subunits were found to co-precipitate, consistent with low levels of the GFP fusions assembling with endogenous CCT subunits (Fig. 2c). The sucrose gradient profiles were then analyzed by densitometry. The endogenous CCT $\delta$ profile is consistent with most of CCT $\delta$ being incorporated in the oligomer (Fig. 2d, top panel). In addition to the B16F1 cells 
Fig. 2 The V390F mutation in CCT反 may promote CCT oligomerization. a Schematic representation of experimental procedures for analysis of constructs in $\mathrm{B} 16 \mathrm{~F} 1$ cells and illustration of the GFP and myctag positions. b Representative sucrose gradient profiles of endogenous CCT $\delta$, GFP-CCT $\delta$, GFP-CCT $\delta^{\mathrm{V} 390 \mathrm{~F}}, \mathrm{CCT} \delta$-myc, and $\mathrm{CCT} \delta^{\mathrm{V} 390 \mathrm{~F}}$-myc. c Immunoprecipitation of the CCT oligomer-containing fractions using anti-GFP nanobodies analyzed by SDS PAGE and silver staining. The GFP-CCT fusions are marked with an asterisk and the position of endogenous CCT subunits indicated with brackets. d Intensity analysis for three experiments as shown in $\mathbf{b}$. Percent of intensity for each fraction was compared to the same set of transfections (GFP$\mathrm{CCT} \delta^{\mathrm{V} 390 \mathrm{~F}}$ to GFP-CCT $\delta$, and $\mathrm{CCT}^{\mathrm{V} 390 \mathrm{~F}}$-myc to CCT $\delta$-myc). Student $t$-test is used where significant when $* p<0.05, * *$ $p<0.01, n=3$

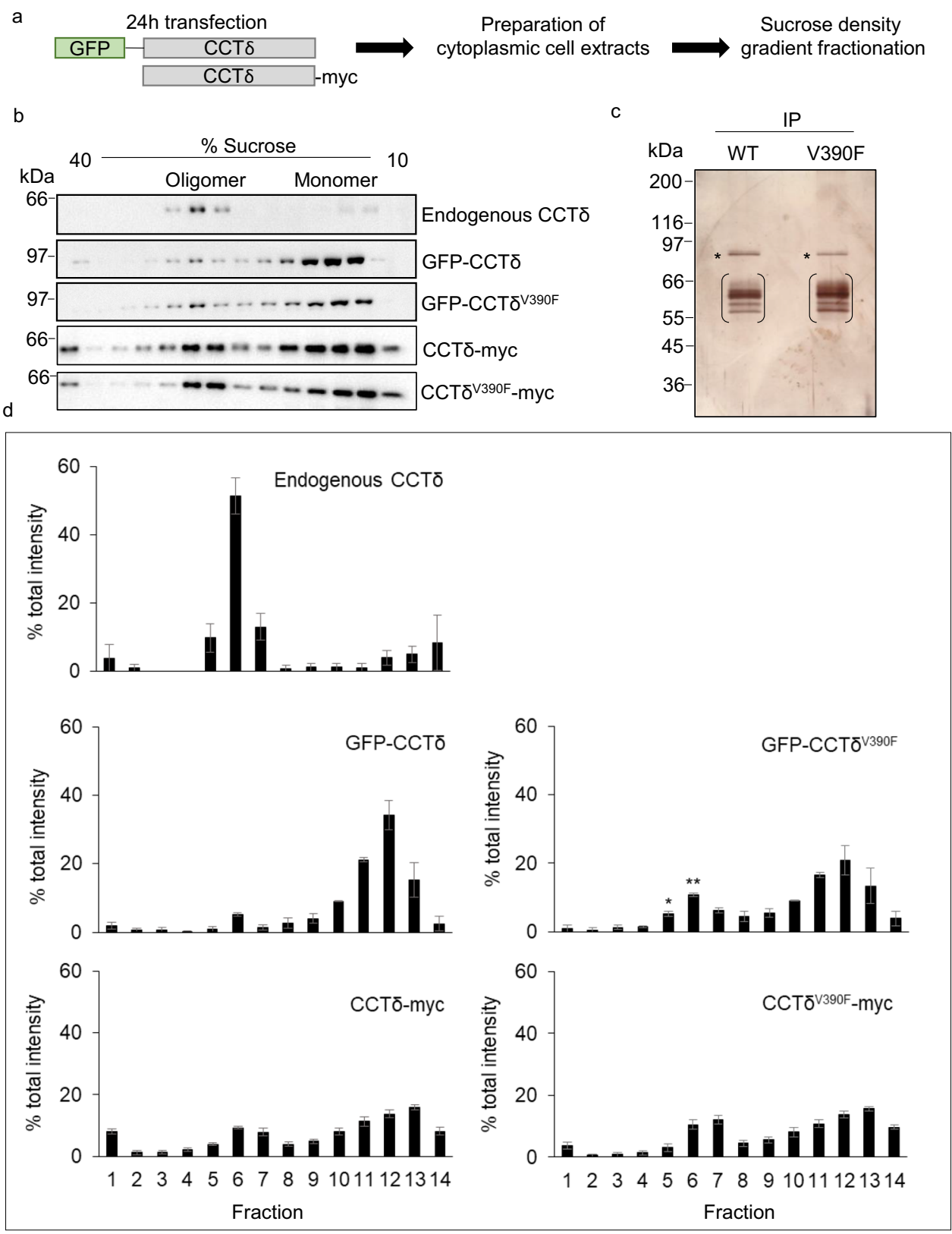

shown here, profiles of endogenous subunits have also been examined in the BE colon carcinoma cell line (Grantham et al. 2006) and balb3T3 mouse fibroblast cell line (Brackley and Grantham 2010) where the oligomeric form is abundant. As expected, the addition of a GFP-tag to the N-terminus of CCT $\delta$ hindered GFP-CCT $\delta$ oligomerization and most of GFP-CCT $\delta$ migrated in the lighter fractions of the sucrose gradient (Fig. 2d, middle left panel). However, in comparison to GFP-CCT $\delta$, GFP-CCT $\delta^{\mathrm{V} 390 \mathrm{~F}}$ was significantly enriched in the CCT oligomer-containing fractions (Fig. 2d, middle right panel). Thus, a single amino acid substitution, from valine to phenylalanine, and thereby the addition of an aromatic side chain in CCT $\delta$ can override the robust method of hindering oligomer incorporation by the addition of a
GFP-tag to the N-terminus. We then assessed the effect of the V390F substitution when expressed as CCT $\delta^{\mathrm{V} 390 \mathrm{~F}}$-myc and observed a slight, although not statistically significant, enhancement of $\mathrm{CCT} \delta^{\mathrm{V} 390 \mathrm{~F}}$-myc in the oligomeric fractions (Fig. 2d, lower panels). The Western blot signals for endogenous CCT $\delta$ and the GFP and myc constructs from fractions corresponding to the oligomer and monomer peaks in the sucrose gradient constructs were combined and assessed as a percentage of the total signal (Table 1). This analysis reflects that of the individual sucrose gradient profiles shown in Fig. 2d. As CCT $\delta$-myc is readily able to oligomerize, differences between the mutant and wild-type constructs may be less apparent, as enhanced oligomerization by V390F may then be limited by the levels of the endogenous subunits. 
Table 1 Western blot signals corresponding to the oligomer and monomer fractions were calculated as a percentage of the total signal across the gradient profile. In all cases, the oligomer was considered to be in fractions 5-7 and the free monomer pool was considered to be fractions $12-14$ for endogenous and myc-tagged, and 11-13 for GFP fusions. Standard error of the mean $( \pm)$ is calculated from the percentage of the total signal for oligomer or monomer fractions (1113 or $12-14$ and $5-7$, respectively) from three individual experiments $(n=3)$

\begin{tabular}{|c|c|c|}
\hline & Oligomer & Monomer \\
\hline Endogenous CCT反 & $73.97 \% \pm 6.14$ & $17.05 \% \pm 10.3$ \\
\hline GFP-CCT $\delta$ & $7.49 \% \pm 1.76$ & $70.75 \% \pm 1.72$ \\
\hline GFP-CCT $\delta^{\mathrm{V} 390 \mathrm{~F}}$ & $22.17 \% \pm 1.17$ & $50.77 \% \pm 2.37$ \\
\hline СCT $\delta$-myc & $21.10 \% \pm 1.93$ & $37.94 \% \pm 2.89$ \\
\hline $\mathrm{CCT} \delta^{\mathrm{V} 390 \mathrm{~F}}-\mathrm{myc}$ & $25.57 \% \pm 1.24$ & $39.05 \% \pm 0.73$ \\
\hline
\end{tabular}

\section{The V390F substitution abrogates the binding of CCT $\delta$ to $150^{\text {Glued }}$}

When levels of CCT $\delta$ monomer are increased by transfection, CCT $\delta$ translocates to the plasma membrane and there is an induction of actin containing cell surface protrusions, which we refer to as a 'protrusion phenotype' (Spiess et al. 2015). This phenotype was used to study functions of monomeric CCT $\delta$ and we found that the $\mathrm{p} 150^{\text {Glued }}$ component of the dynactin complex binds to CCT (Echbarthi et al. 2018). Together with the transmembrane protein dynAP, $\mathrm{p} 150^{\mathrm{Glued}}$ is required for the formation of the cell surface protrusions by creating an inward movement of the plasma membrane along microtubules (Echbarthi et al. 2018 and illustrated in Fig. 3a).

Two points of mutations within $\mathrm{CCT} \delta$, D104E and G357D, prevent the formation of the protrusion phenotype and do not translocate to the plasma membrane (Spiess et al. 2015). D104E is an ATP-binding pocket mutation, and GFP-CCT $\delta^{\text {D104E }}$ has been shown to bind to $\mathrm{p} 150^{\text {Glued }}$, but does not induce cell surface protrusions, whilst the apical domain mutation G357D abolishes binding to $\mathrm{p} 150^{\text {Glued }}$ and therefore cells expressing GFP-CCT $\delta^{\mathrm{G} 357 \mathrm{D}}$ do not display the protrusion phenotype (Echbarthi et al. 2018). Consistent with $\mathrm{p} 150^{\text {Glued }}$ binding to the dynein motor and mediating minus end-directed microtubule transport, we demonstrated that the protrusion phenotype does not form when cells are treated with the microtubule-depolymerizing drug, nocodazole (Echbarthi et al. 2018). We have therefore hypothesized that CCT $\delta$ binds via its apical domain to $\mathrm{p} 150^{\mathrm{Glued}}$ and, via its ATPase activity, may enhance the binding of $\mathrm{p} 150^{\text {Glued }}$ to dynAP at the plasma membrane (Echbarthi et al. 2018).

To study if increased expression of CCT $\delta$ harbouring the $\mathrm{V} 390 \mathrm{~F}$ mutation can induce the protrusion phenotype, B16F1 cells were transfected with GFP-CCT $\delta$ or GFP$\mathrm{CCT} \delta^{\mathrm{V} 390 \mathrm{~F}}$ and scored as having high, low, or no protrusion phenotype upon analysis by wide-field microscopy
(Fig. 3b). Quantification from three biological replicates demonstrates there is a substantial reduction in the number of cells with a protrusion phenotype in GFP-CCT $\delta^{\mathrm{V} 390 \mathrm{~F}}$ expressing cells compared to cells expressing GFP-CCT $\delta$ (Fig. 3c). We then assessed if GFP-CCT $\delta^{\mathrm{V} 390 \mathrm{~F}}$ is able to interact with $\mathrm{p} 150^{\text {Glued }}$. Immunoprecipitation using GFP nanobody beads following co-transfection of $\mathrm{p} 150^{\mathrm{Glued}}$-myc with GFP-CCT $\delta$, GFP-CCT $\delta^{\mathrm{V} 390 \mathrm{~F}}$, or GFP-CCT $\delta^{\mathrm{G} 357 \mathrm{D}}$ (as a negative control) indicated no binding of GFP-CCT $\delta^{\mathrm{V} 390 \mathrm{~F}}$ to p150 ${ }^{\text {Glued }}$-myc (Fig. $3 \mathrm{~d}$ and e), consistent with the expression of GFP-CCT $\delta^{\mathrm{V} 390 \mathrm{~F}}$ not inducing a protrusion phenotype. It is important to note that whilst some GFP-CCT $\delta^{\mathrm{V} 390 \mathrm{~F}}$ will be incorporated into the CCT oligomer, a substantial amount remains monomeric (Fig. 2d and Table 1); thus, it is unlikely that the loss of interaction with $\mathrm{p} 150^{\text {Glued }}$ is due to limiting levels of monomeric GFP-CCT $\delta^{\mathrm{V} 390 \mathrm{~F}}$.

Since $\mathrm{CCT} \delta^{\mathrm{V} 390 \mathrm{~F}}$ has a wild-type apical domain, we speculate that either V390 contributes to binding to $\mathrm{p} 150^{\mathrm{Glued}}$, or that the V390F substitution affects the conformation of the CCT $\delta$ apical domain. The latter possibility is consistent with the position of V390 being at the boundary between the apical domain and the flexible intermediate domain, which is responsible for inducing conformational changes from the equatorial domain to the apical domain.

\section{Assessment of cells expressing CCT mutations using chemotaxis assays with and without GrowDex ${ }^{\circledR}$ 3D matrix}

Levels of GFP-CCT $\delta$ have previously been shown to affect cell migration, where cells having a moderate expression level of GFP-CCT $\delta$ show increased migration in wound healing assays in comparison to cells expressing GFPCCT $\beta$, GFP-CCT $\delta^{\mathrm{G} 357 \mathrm{D}}$, or GFP-CCT $\delta^{\text {D104E }}$ (Echbarthi et al. 2018). As this increased, directed cell migration is observed only where increased levels of monomeric CCT $\delta$ can bind to $\mathrm{p} 150^{\text {Glued }}$ and have a functional ATPase activity,

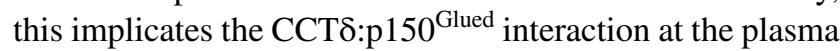
membrane in affecting cell migration (Echbarthi et al. 2018).

To extend our previous studies, here we used a Boyden chamber trans-well assay where we incorporate a layer of gel matrix and use laminin as a chemoattractant to assess the migration of cells in a more 3D environment using B16F1 cells transfected with GFP-CCT $\delta$, GFP-CCT $\delta^{\mathrm{V} 390 \mathrm{~F}}$, GFP$\mathrm{CCT} \delta^{\mathrm{D} 104 \mathrm{E}}$, or GFP-CCT $\delta^{\mathrm{G} 357 \mathrm{D}}$.

Cells were either seeded directly onto the Boyden chamber membrane or onto a layer of GrowDex ${ }^{\circledR}$ cellulosecontaining hydrogel (Fig. 4a). The use of GrowDex ${ }^{\circledR}$ is beneficial as it enables us to use laminin as a chemoattractant, compared to animal-based gels, which already contain laminin. Initially, we confirmed that cells only translocated through the membrane of the Boyden chamber in the presence of laminin (Fig. 4b). We then scored the numbers of 


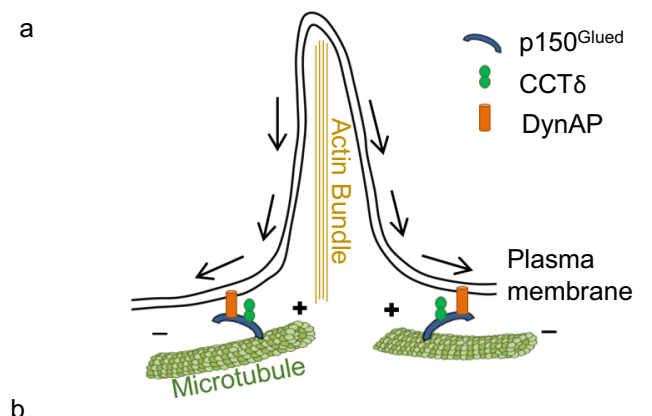

b
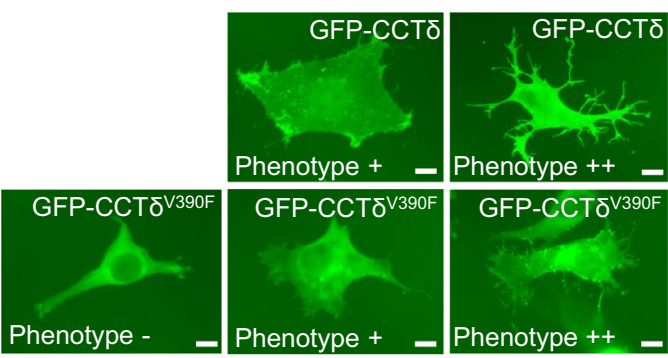

d
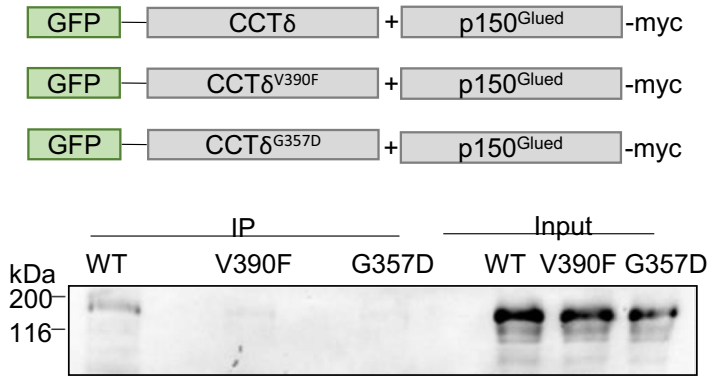

C

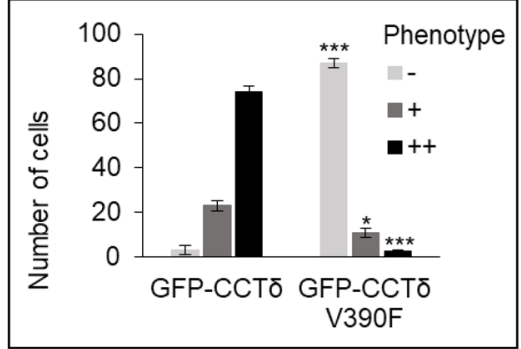

e

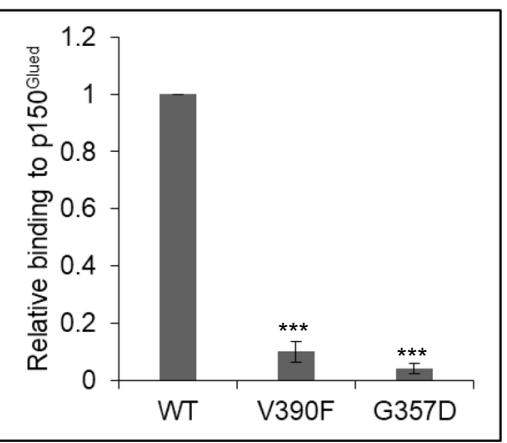

Fig. 3 GFP-CCT $\delta^{\mathrm{V} 390 \mathrm{~F}}$ does not bind to $\mathrm{p} 150^{\mathrm{Glued}}$. a Illustration of an inward movement of the plasma membrane induced by monomeric CCT $\delta$ binding to $150^{\text {Glued }}$ as described in Echbarthi et al. (2018). b Wide-field microscopy images of B16F1 cells transfected with GFPCCT $\delta$ or GFP-CCT $\delta^{\mathrm{V} 390 \mathrm{~F}}$. Scale bars correspond to $10 \mu \mathrm{m}$. c Quantification of cells scored for having no $(-)$, weak $(+)$, or strong $(++)$ protrusion phenotype. Three biological replicates were performed and 100 cells from each counted. The graph shows the mean from the three experiments and Student $t$-test was used comparing WT to V390F $(*<0.05$ and $* * *<0.001)$. d The combinations of constructs

transfected cells translocating through the membrane in the presence and absence of GrowDex ${ }^{\circledR}$. Representative images of GFP-CCT $\delta$ expressing cells seeded directly onto the Boyden chamber or onto a layer of GrowDex ${ }^{\circledR}$ are shown in Fig. 4c. Cells included in the analysis were those that had completely translocated through the pores of the membrane and those in the process of translocating through the membrane.

Consistent with GrowDex ${ }^{\circledR}$ providing a substantial 3D matrix, there is a statistically significant reduction the number of GFP-CCT $\delta$ expressing cells able to migrate through the trans-well membrane in the presence GrowDex ${ }^{\circledR}$ 3D matrix compared to GFP-CCT $\delta$ expressing cells seeded in used for immunoprecipitation are illustrated. Immunoprecipitation with anti-GFP nanobodies from lysates of cells co-transfected with p150 ${ }^{\text {Glued }}$-myc and GFP-CCT 8 , GFP-CCT $\delta^{\mathrm{V} 390 \mathrm{~F}}$, or GFP-CCT ${ }^{\mathrm{G} 357 \mathrm{D}}$ analyzed by Western blotting using anti-myc antibody. e Analysis of three biological replicates of $\mathbf{d}$ are shown. For each individual experiment, the signal from the IP is normalized to input and WT relative binding is set to 1 . The relative binding for the two mutations is compared to WT. The graph shows the mean of the three experiments. Student $t$-test was used to compare the mutations to WT $(* * *<0.001), n=3$

the absence of GrowDex ${ }^{\circledR}$ (Fig. 4d). For cells expressing GFP-CCT $^{\mathrm{V} 390 \mathrm{~F}}$, GFP-CCT $\delta^{\mathrm{D} 104 \mathrm{E}}$, or GFP-CCT $\delta^{\mathrm{G} 357 \mathrm{D}}$, there was no statistical difference in the numbers of cells translocating through the membrane in the presence and absence of GrowDex $\AA$, although the overall trend indicated a slight decrease in the number of translocating cells in the presence GrowDex ${ }^{\circledR}$ (Fig. 4d).

Next, to compare chemotaxis migration in the absence of GrowDex ${ }^{\circledR}$ between GFP-CCT $\delta$ and GFP-CCT $\delta^{\mathrm{V} 390 \mathrm{~F}}$, GFP-CCT $\delta^{\text {D104E }}$, and GFP-CCT $\delta^{\mathrm{G} 357 \mathrm{D}}$, the numbers of cells translocating through the membrane for each of the mutations were compared to GFP-CCT $\delta$ transfected cells. The mean number of translocating cells was compared between 
Fig. 4 Effects of CCT $\delta$ mutations on chemotaxis migration. a Schematic overview of the experimental chemotaxis procedure where cells migrate through a porous membrane towards a chemoattractant with and without a layer of $0.3 \%$ GrowDex ${ }^{\circledR}$ matrix. b Wide-field microscopy images of cells migrating through a Boyden chamber trans-well membrane with and without laminin. c Representative wide-field microscopy images showing the experimental procedure described in $\mathbf{a} . \mathbf{d}$ Comparison of the amount of cells migrating towards laminin through the Boyden chamber trans-well membrane compared to cells migrating towards laminin through the membrane after they have migrated through 0.3\% GrowDex ${ }^{\circledR}$ 3D matrix layer. Student $t$-test is used where significant when ${ }^{*} p<0.05, * * p<0.01$. e Chemotaxis migration in the absence of GrowDex ${ }^{\circledR}$ where the ratio between cells expressing GFP$\mathrm{CCT}^{\mathrm{V} 390 \mathrm{~F}}$, GFP-CCT $\delta^{\mathrm{D} 104 \mathrm{E}}$ and GFP-CCT $\delta^{\mathrm{G} 357 \mathrm{D}}$ and cells expressing GFP-CCT $\delta$ at three time points is calculated. GFP-CCT $\delta$ is set to 1 . f Ratio between chemotaxis migration as described above for cells migrating through $0.3 \%$ GrowDex ${ }^{\circledR}$. For each set of experiments in $\mathbf{d}, \mathbf{e}$, and f, $n$ is between 2 and 5 where statistical analysis only has been performed where $n \geq 3$. Scale bars correspond to $100 \mu \mathrm{m}$ a
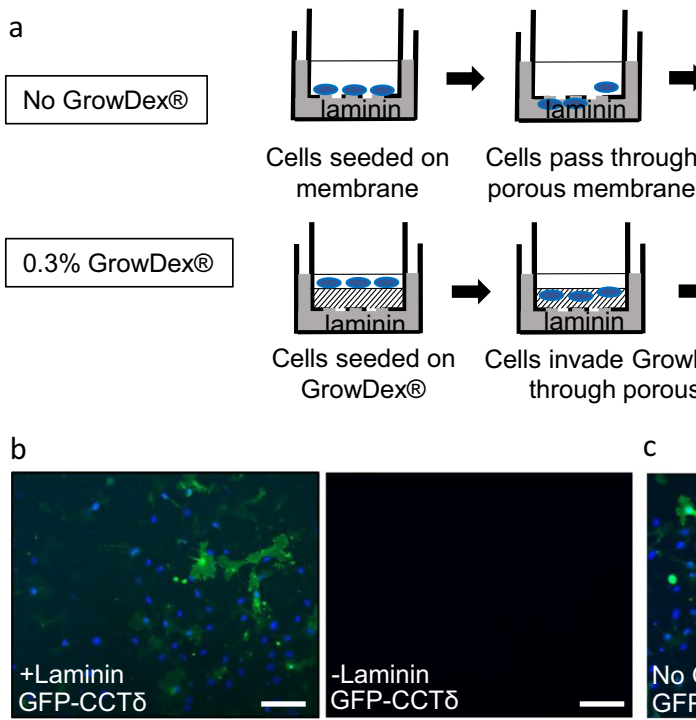

Cells seeded on Cells pass through Translocated cells membrane porous membrane analysed by microscopy

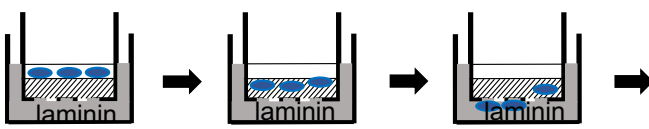

Cells seeded on Cells invade GrowDex® and pass GrowDex® through porous membrane

Translocated cells analysed by microscopy

C
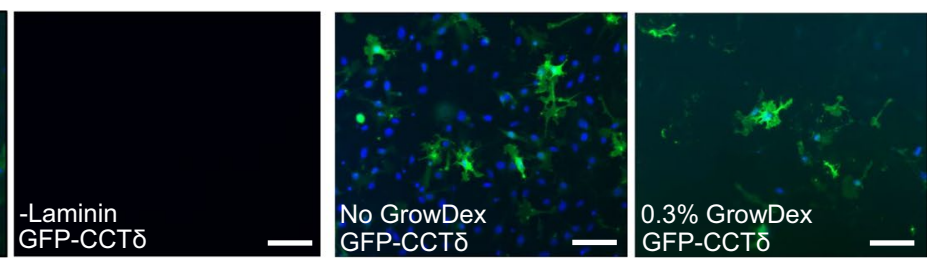

d
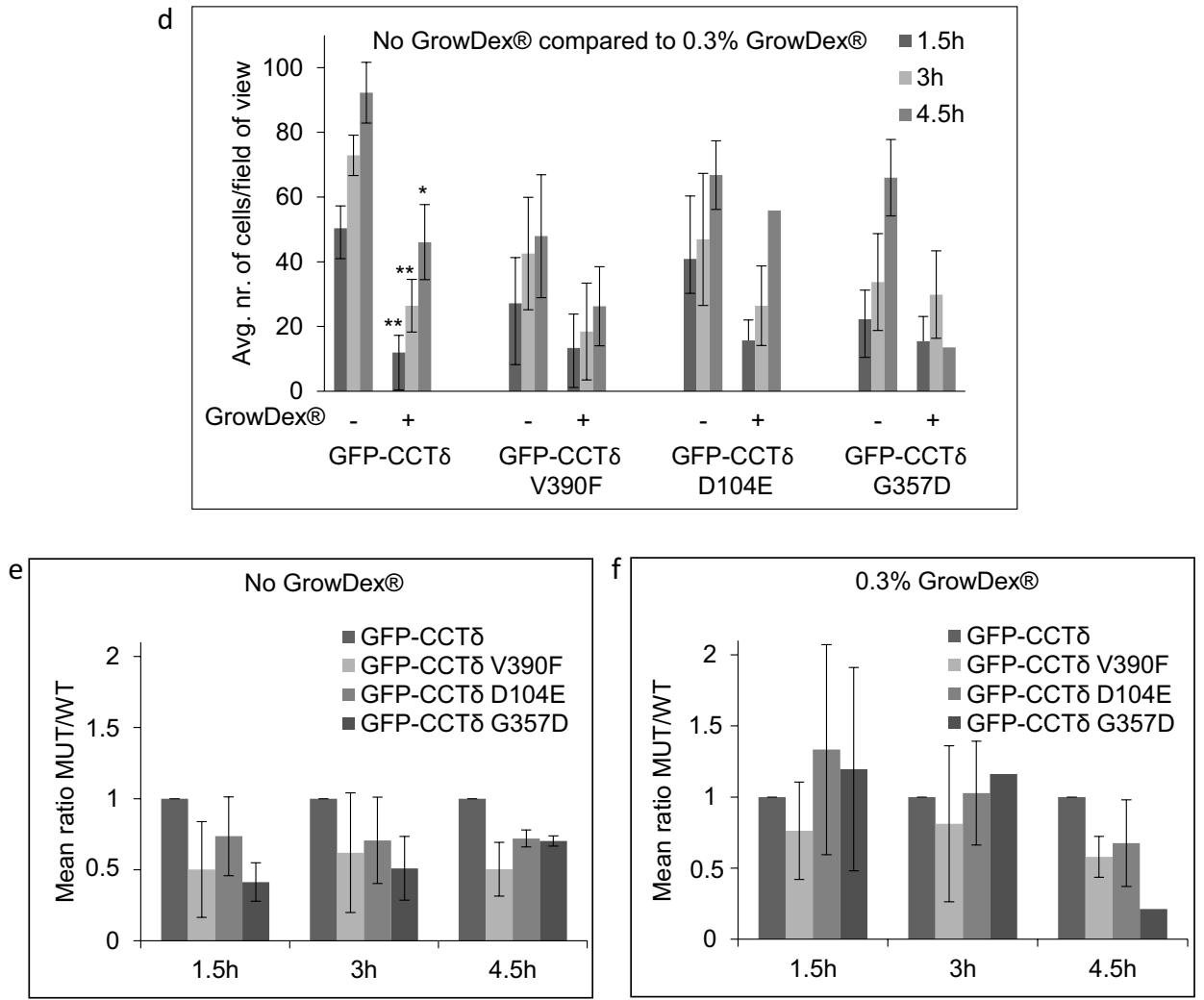

GFP-CCT $\delta$ and GFP-CCT $\delta^{\mathrm{V} 390 \mathrm{~F}}$, GFP-CCT $\delta^{\mathrm{D} 104 \mathrm{E}}$, and GFP-CCT $\delta^{\mathrm{G} 357 \mathrm{D}}$, and the ratio between GFP-CCT $\delta$ and GFP-CCT $\delta^{\mathrm{V} 390 \mathrm{~F}}$, GFP-CCT $\delta^{\mathrm{D} 104 \mathrm{E}}$, and GFP-CCT $\delta^{\mathrm{G} 357 \mathrm{D}}$, where GFP-CCT $\delta$ is set to 1 , was calculated (Fig. 4e). Although not statistically significant, there is a trend of the chemotaxis movement being reduced in cells expressing GFP-CCT $\delta^{\mathrm{V} 390 \mathrm{~F}}$, GFP-CCT $\delta^{\mathrm{D} 104 \mathrm{E}}$, or GFP-CCT $\delta^{\mathrm{G} 357 \mathrm{D}}$ in comparison to cells expressing GFP-CCT $\delta$ (Fig. 4e). In contrast, when the same experiment was performed with a layer of $0.3 \%$ GrowDex ${ }^{\circledR}$, a reduction in the number of migrating cells for GFP-CCT $\delta^{\mathrm{V} 390 \mathrm{~F}}$, GFP-CCT $\delta^{\mathrm{D} 104 \mathrm{E}}$, or GFP-CCT $\delta^{\text {G357D }}$ expressing cells compared to GFP-CCT $\delta$ during chemotaxis up to $3 \mathrm{~h}$ was not observed (Fig. 4f).

To confirm that the differences observed were not a consequence of transfection efficiency, we calculated the transfection efficiencies and observed that this is in fact lower for GFP-CCT $\delta$ compared to that of GFP$\mathrm{CCT}^{\mathrm{V} 390 \mathrm{~F}}$, GFP-CCT $\delta^{\mathrm{D} 104 \mathrm{E}}$, and GFP-CCT $\delta^{\mathrm{G} 357 \mathrm{D}}$. For example, in one biological replicate, the transfection efficiencies were GFP-CCT $\delta=65 \%$, GFP-CCT $\delta^{\mathrm{V} 390 \mathrm{~F}}=75 \%$, 
GFP-CCT $\delta^{\mathrm{D} 104 \mathrm{E}}=72 \%$, and GFP-CCT $\delta^{\mathrm{G} 357 \mathrm{D}}=68 \%$. The reduction in chemotaxis movement here is consistent with the observations of Echbarthi et al. (2018), where cells expressing GFP-CCT $\delta^{\mathrm{D} 104 \mathrm{E}}$ or GFP-CCT $\delta^{\mathrm{G} 357 \mathrm{D}}$ migrated more slowly in a wound healing assay compared to cells expressing GFP-CCT $\delta$.

None of the mutations used here are able to induce the protrusion phenotype, which we consider to be a consequence of CCT $\delta$ monomer binding to $150^{\text {Glued }}$ and the ATPase activity of CCT $\delta$ inducing translocation to the plasma membrane (Echbarthi et al. 2018). This would suggest that the higher levels of cell migration observed for cells expressing GFP-CCT $\delta$ could be a consequence of increased interactions with $\mathrm{p} 150^{\mathrm{Glued}}$. The observations here that GFPCCT $\delta$ is not faster through $0.3 \%$ GrowDex ${ }^{\circledR}$ compared to the three mutants suggest that the interaction between monomeric CCT $\delta$ and $\mathrm{p} 150^{\text {Glued }}$ could potentially have a greater impact on certain types of cell motility. It is also possible that there is a difference in how the cells respond to laminin between the CCT $\delta$ constructs tested.

\section{Discussion}

Here, we have assessed the $\mathrm{CCT} \delta^{\mathrm{V} 390 \mathrm{~F}}$ mutation with regard to oligomer assembly and monomeric function. We demonstrate that this mutation may promote assembly of the CCT oligomer, and as a monomer is unable to bind $\mathrm{p} 150^{\mathrm{Glued}}$. Thus, there are potentially two distinct consequences for cells harbouring the V390F mutation: increased levels of CCT oligomer and loss of $\mathrm{p} 150^{\text {Glued }}$-mediated CCT 8 monomer function (Fig. 5). The former may increase the folding capacity of the cell, whilst the latter will potentially reduce the possibility of certain types of cell migration. More assembled CCT oligomer would support an enhanced growth rate via the folding of actin and tubulin and other CCT folding substrates required for cell cycle progression, such as Cdc20 (Camasses et al. 2003). Indeed, CCT levels have previously been linked to cell growth (Yokota et al. 1999), and when CCT levels are depleted by siRNA, a decrease in cell growth is observed (Grantham et al. 2006).

CCT subunits can undergo gain of function/amplification in cancer and have, for example, been suggested to support breast cancer cell growth (Ghozlan et al. 2021; Xu et al. 2021). However, the effects of mutations of CCT subunits are less clear. It is possible that some mutations, such as the V390F substitution reported here, affect oligomerization and will thus have the potential effect in the cell in the same way as an increase in CCT expression levels and lead to an increased folding capacity/cell growth rates. The extent of CCT oligomer assembly will always be limited by the abundance of the other subunits, as they all exist in equal amounts in the assembled oligomer. Also, enhancement of

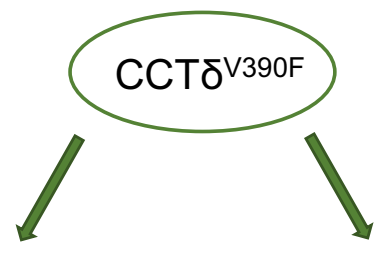

Increased
CCT oligomer

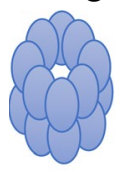

$\downarrow$

Enhanced folding
capacity?

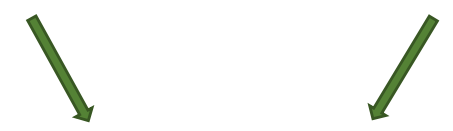

Altered profile of oligomer
/monomer functions

Fig. 5 Consequences of CCT $\delta$ V390F mutation. Schematic figure to show that V390F mutation in CCT $\delta$ could result in two separate effects in the cell, increased CCT oligomer, and loss of CCT $\delta$ monomer function. Affecting CCT assembly or monomer-specific interactions would both result in an altered profile of oligomer and monomer functions

oligomerization will potentially deplete the CCT monomer pool, reducing the availability of free subunits to perform monomeric-specific functions, such as $\mathrm{CCT} \varepsilon$ binding to MRTF-A (Elliott et al. 2015). Furthermore, point mutations may interrupt specific binding interactions, as is the case for the V390F mutation with regard to $\mathrm{p} 150^{\mathrm{Glued}}$ binding. Thus, the effects of mutations and changes in CCT subunit expression may have complex consequences for the cell.

Mutations affecting assembly will have the potential to not only increase the folding capacity of the cell, but may also influence the availability of CCT oligomer for functions beyond folding such as modulating STAT3 activity (Vallin et al. 2021) and gelsolin activity (Svanstrom and Grantham 2016) and promoting the assembly of the von Hippel-Lindau tumour suppressor protein and Elongins (Melville et al. 2003).

Mutations in CCT subunits and changes in expression levels are not only relevant for cancer cell biology; for example, Brehme et al. (2014) report reductions in CCT 
expression during ageing and some neurodegenerative diseases. Furthermore, the $\mathrm{C} 450 \mathrm{Y}$ mutation in CCT 8 associated with hereditary sensory neuropathies may display reduced assembly (Sergeeva et al. 2014). Thus, the consequences of mutations in CCT subunits and changes in CCT expression levels, together with how CCT assembly is regulated, remain important questions for understanding the role of CCT in health and disease.

\section{Methods}

\section{Cell culture}

B16F1 cells were maintained in complete DMEM media (DMEM media (GIBCO Life Technologies) supplemented with $100 \mathrm{U} / \mathrm{ml}$ penicillin-streptomycin (GIBCO Life Technologies), $2.5 \mu \mathrm{g} / \mathrm{ml}$ Plasmocin (InvivoGen), and $10 \%$ heatinactivated FBS (Invitrogen)) at $37^{\circ} \mathrm{C}, 5 \% \mathrm{CO}_{2}$.

\section{Transfection}

GFP-CCT $\delta$, GFP-CCT $\delta^{\text {D104E }}$ and GFP-CCT $\delta^{\mathrm{G} 357 \mathrm{D}}$ plasmid preparation is described by Spiess et al. (2015) and p150 Glued plasmid preparation is described by Echbarthi et al. (2018). The GFP-CCT $\delta^{\mathrm{V} 390 \mathrm{~F}}$ plasmid was prepared using QuickChange $^{\mathrm{TM}}$ site-directed mutagenesis cloning method using GFP-CCT $\delta$ as the template. B16F1 cells were transfected using Opti-MEM (GIBCO Life Technologies) together with Lipofectamine 2000 (Invitrogen).

\section{Sucrose density gradient fractionation}

One day post-transfection, cells were detached from the cell culture dish by addition of $1 \mathrm{mM}$ EDTA in PBS and washed twice in ice-cold PBS. Cells were then lysed in ice-cold lysis buffer (50 mM HEPES pH 7.2, 90 mM KCl, 0.5\% IGEPAL, and 1/500 mammalian protease inhibitor (Sigma-Aldrich)) and post-nuclear supernatants obtained by centrifugation at $7000 \mathrm{rpm}$ for $5 \mathrm{~min}$ at $4{ }^{\circ} \mathrm{C}$ in a benchtop centrifuge. Samples were loaded onto sucrose gradients containing a gradient of $40-10 \%$ sucrose in $50 \mathrm{mM}$ HEPES $\mathrm{pH} 7.2$, $90 \mathrm{mM} \mathrm{KCl}$. Samples were then centrifuged at $4{ }^{\circ} \mathrm{C}$ for $18 \mathrm{~h}$ at 85,000 $\mathrm{g}$ using a Beckman SW55 Ti rotor. Fourteen equal volume fractions were collected and resolved by SDS PAGE, before being transferred to a nitrocellulose membrane and analyzed by Western blotting.

\section{Immunofluorescence}

On the day before transfection, B16F1 cells were seeded on glass coverslips that were pre-coated with laminin (SigmaAldrich). Cells were transfected on the glass coverslips, and the day after transfection, cells were washed once in complete PBS (supplemented with $1 \mathrm{mM} \mathrm{CaCl}_{2}$ and $0.5 \mathrm{mM}$ $\mathrm{MgCl}_{2}$ ) and incubated for $10 \mathrm{~min}$ in $4 \%$ formaldehyde in complete PBS. Fixed cells were washed three times in PBS and with a final wash in MilliQ water before being mounted onto microscope slides using ProLong®Gold (Invitrogen). Wide-field images were taken using a Zeiss Axioplan microscope with AxioVision software.

\section{Immunoprecipitation}

For immunoprecipitation, B16F1 cells were transfected, and the day after transfection, post-nuclear supernatants were prepared as described above. An additional centrifugation at $13,000 \mathrm{rpm}$ for $2 \mathrm{~min}$ at $4{ }^{\circ} \mathrm{C}$ in a benchtop centrifuge was performed before incubation with GFP-trap ${ }^{\circledR} \_$A beads (Chromotek) for $1 \mathrm{~h}$ at $4{ }^{\circ} \mathrm{C}$ on a rotating wheel at $9 \mathrm{rpm}$. After incubation, beads were washed three times in lysis buffer and vacuum-dried before the addition of $1 \times$ SDS sample buffer. Samples were resolved by SDS PAGE, before being transferred to a nitrocellulose membrane and analyzed by Western blotting.

\section{Boyden chamber migration}

B16F1 cells were grown in normal cell culture conditions and transfected as described above. The next day, cells were detached as described above and diluted to give a final concentration of $130 \times 10^{4}$ cells $/ \mathrm{ml}$. The Boyden chamber membrane inserts (TREVIGEN) were hydrated by addition of $100 \mu \mathrm{l}$ DMEM media and were incubated in the cell culture incubator $\left(37^{\circ} \mathrm{C}, 5 \% \mathrm{CO}_{2}\right)$ for at least $30 \mathrm{~min}$. GrowDex ${ }^{\circledR}$ (UPM Biomedicals) was dispensed from the syringe and weighed. DMEM media was added to give a final concentration of $0.3 \% \mathrm{w} / \mathrm{v}$ GrowDex ${ }^{\circledR}$ and mixed for $90 \mathrm{~s}$ to give an even distribution of GrowDex ${ }^{\circledR}$ in the tube. For membranes used for 3D migration studies, the $100 \mu \mathrm{l}$ of DMEM incubated with the membrane was replaced by $100 \mu \mathrm{l} 0.3 \%$ GrowDex ${ }^{\circledR}$. Fifty microliters of cell suspension $\left(130 \times 10^{4}\right.$ cells $\left./ \mathrm{ml}\right)$ were added on top of the $100 \mu \mathrm{l}$ DMEM or GrowDex ${ }^{\circledR}$ and moved to a well containing a 1/40 dilution of laminin in DMEM (Sigma-Aldrich L2020) to form the attractant gradient. Inserts were incubated in the cell culture incubator for $1.5,3$, or $4.5 \mathrm{~h}$ before fixing in $4 \%$ formaldehyde in complete PBS for $10 \mathrm{~min}$. Inserts were washed 3 times in PBS, and the cells remaining on the upper side of the membrane were cleaned away using a cotton swab. Cells were then stained with $1 \mu \mathrm{g} / \mathrm{ml}$ DAPI (Thermo Scientific) and washed 3 times in PBS, and then, membranes were cut out from the insert and mounted onto a glass microscope slide using ProLong ${ }^{\circledR G o l d ~(I n v i t r o g e n) . ~ W i d e-f i e l d ~ i m a g e s ~}$ were taken using a Zeiss Axioplan microscope with AxioVision software. 


\section{Sequence alignment}

Sequences for mouse and human CCT subunits were obtained from UniProt and sequences were aligned using BLAST to compare the location of the V390 position in CCTS to other subunits. The sequence of the thermosome was also aligned using BLAST, and the position corresponding to the CCTS mutation mapped onto the structure of thermosome alpha chain (obtained from PDB: 1A6D) by using PyMol.

Supplementary Information The online version contains supplementary material available at https://doi.org/10.1007/s12192-021-01237-x.

Acknowledgements We thank Dr. Kerryn Elliott for input on COSMIC and Dr. Meriem Echbarthi for help with Boyden chamber assays.

Author contribution JV performed all experiments, analyzed the data, developed the project, and wrote the manuscript. JG analyzed the data, developed the project, and wrote the manuscript.

Funding Open access funding provided by University of Gothenburg. We acknowledge funding support from The Swedish Cancer Society (to JG), Assar Gabrielssons Fund (to JV and JG), and the University of Gothenburg.

Availability of data and material Available on request.

Code availability Not applicable.

\section{Declarations}

Ethics approval Not applicable.

Consent to participate The authors agree to this.

Consent for publication The authors agree to this.

Conflict of interest The authors declare no competing interests.

Open Access This article is licensed under a Creative Commons Attribution 4.0 International License, which permits use, sharing, adaptation, distribution and reproduction in any medium or format, as long as you give appropriate credit to the original author(s) and the source, provide a link to the Creative Commons licence, and indicate if changes were made. The images or other third party material in this article are included in the article's Creative Commons licence, unless indicated otherwise in a credit line to the material. If material is not included in the article's Creative Commons licence and your intended use is not permitted by statutory regulation or exceeds the permitted use, you will need to obtain permission directly from the copyright holder. To view a copy of this licence, visit http://creativecommons.org/licenses/by/4.0/.

\section{References}

Amit M, Weisberg SJ, Nadler-Holly M, McCormack EA, Feldmesser E, Kaganovich D, Willison KR, Horovitz A (2010) Equivalent mutations in the eight subunits of the chaperonin CCT produce dramatically different cellular and gene expression phenotypes. $\mathrm{J}$ Mol Biol 401:532-543

Brackley KI, Grantham J (2010) Subunits of the chaperonin CCT interact with F-actin and influence cell shape and cytoskeletal assembly. Exp Cell Res 316:543-553

Brackley KI, Grantham J (2011) Interactions between the actin filament capping and severing protein gelsolin and the molecular chaperone CCT: evidence for nonclassical substrate interactions. Cell Stress Chaperones 16:173-179

Brehme M, Voisine C, Rolland T, Wachi S, Soper JH, Zhu Y, Orton K, Villella A, Garza D, Vidal M, Ge H, Morimoto RI (2014) A chaperome subnetwork safeguards proteostasis in aging and neurodegenerative disease. Cell Rep 9:1135-1150

Camasses A, Bogdanova A, Shevchenko A, Zachariae W (2003) The CCT chaperonin promotes activation of the anaphase-promoting complex through the generation of functional Cdc20. Mol Cell 12:87-100

Collier MP, Moreira KB, Li KH, Chen YC, Itzhak D, Samant R, Leitner A, Burlingame A, Frydman J (2021) Native mass spectrometry analyses of chaperonin complex TRiC/CCT reveal subunit $\mathrm{N}$-terminal processing and re-association patterns. Sci Rep 11:13084

Echbarthi M, Vallin J, Grantham J (2018) Interactions between monomeric CCTdelta and p150(Glued): a novel function for CCTdelta at the cell periphery distinct from the protein folding activity of the molecular chaperone CCT. Exp Cell Res 370(1):137-149

Elliott KL, Svanstrom A, Spiess M, Karlsson R, Grantham J (2015) A novel function of the monomeric CCTepsilon subunit connects the serum response factor pathway to chaperone-mediated actin folding. Mol Biol Cell 26:2801-2809

Ghozlan H, Showalter A, Lee E, Zhu X, Khaled AR (2021) Chaperonin-containing TCP1 complex (CCT) promotes breast cancer growth through correlations with key cell cycle regulators. Front Oncol 11:663877

Grantham J, Brackley KI, Willison KR (2006) Substantial CCT activity is required for cell cycle progression and cytoskeletal organization in mammalian cells. Exp Cell Res 312:2309-2324

Liou AK, Willison KR (1997) Elucidation of the subunit orientation in CCT (chaperonin containing TCP1) from the subunit composition of CCT micro-complexes. EMBO J 16:4311-4316

Matalon O, Horovitz A, Levy ED (2014) Different subunits belonging to the same protein complex often exhibit discordant expression levels and evolutionary properties. Curr Opin Struct Biol 26:113-120

Melville MW, McClellan AJ, Meyer AS, Darveau A, Frydman J (2003) The Hsp70 and TRiC/CCT chaperone systems cooperate in vivo to assemble the von Hippel-Lindau tumor suppressor complex. Mol Cell Biol 23:3141-3151

Roobol A, Grantham J, Whitaker HC, Carden MJ (1999a) Disassembly of the cytosolic chaperonin in mammalian cell extracts at intracellular levels of K+ and ATP. J Biol Chem 274:19220-19227

Roobol A, Sahyoun ZP, Carden MJ (1999b) Selected subunits of the cytosolic chaperonin associate with microtubules assembled in vitro. J Biol Chem 274:2408-2415

Sergeeva OA, Tran MT, Haase-Pettingell C, King JA (2014) Biochemical characterization of mutants in chaperonin proteins CCT4 and CCT5 associated with hereditary sensory neuropathy. J Biol Chem 289:27470-27480

Showalter AE, Martini AC, Nierenberg D, Hosang K, Fahmi NA, Gopalan P, Khaled AS, Zhang W, Khaled AR (2020) Investigating chaperonin-containing TCP-1 subunit 2 as an essential component of the chaperonin complex for tumorigenesis. Sci Rep 10:798

Spiess M, Echbarthi M, Svanstrom A, Karlsson R, Grantham J (2015) Over-Expression analysis of all eight subunits of the molecular chaperone CCT in mammalian cells reveals a novel function for CCTdelta. J Mol Biol 427:2757-2764 
Sternlicht H, Farr GW, Sternlicht ML, Driscoll JK, Willison K, Yaffe MB (1993) The t-complex polypeptide 1 complex is a chaperonin for tubulin and actin in vivo. Proc Natl Acad Sci U S A 90:9422-9426

Stoldt V, Rademacher F, Kehren V, Ernst JF, Pearce DA, Sherman F (1996) Review: the Cct eukaryotic chaperonin subunits of Saccharomyces cerevisiae and other yeasts. Yeast 12:523-529

Svanstrom A, Grantham J (2016) The molecular chaperone CCT modulates the activity of the actin filament severing and capping protein gelsolin in vitro. Cell Stress Chaperones 21:55-62

Vallin J, Grantham J (2019) The role of the molecular chaperone CCT in protein folding and mediation of cytoskeleton-associated processes: implications for cancer cell biology. Cell Stress Chaperones $24: 17-27$

Vallin J, Cordoba-Beldad CM, Grantham J (2021) Sequestration of the transcription factor STAT3 by the molecular chaperone CCT: a potential mechanism for modulation of STAT3 phosphorylation. J Mol Biol 433(13): 166958

Wang W, Goswami S, Lapidus K, Wells AL, Wyckoff JB, Sahai E, Singer RH, Segall JE, Condeelis JS (2004) Identification and testing of a gene expression signature of invasive carcinoma cells within primary mammary tumors. Can Res 64:8585-8594

Willison KR (2018) The substrate specificity of eukaryotic cytosolic chaperonin CCT. Philos Trans R Soc Lond B Biol Sci 373(1749):20170192

Xu WX, Song W, Jiang MP, Yang SJ, Zhang J, Wang DD, Tang JH (2021) Systematic characterization of expression profiles and prognostic values of the eight subunits of the chaperonin TRiC in breast cancer. Front Genet 12:637887

Yokota S, Yamamoto Y, Shimizu K, Momoi H, Kamikawa T, Yamaoka Y, Yanagi H, Yura T, Kubota H (2001) Increased expression of cytosolic chaperonin CCT in human hepatocellular and colonic carcinoma. Cell Stress Chaperones 6:345-350

Yokota S, Yanagi H, Yura T, Kubota H (1999) Cytosolic Chaperonin Is Up-regulated during Cell Growth. J Biol Chem 274(52) 37070-37078. https://doi.org/10.1074/jbc.274.52.37070

Publisher's note Springer Nature remains neutral with regard to jurisdictional claims in published maps and institutional affiliations. 